\title{
Bilateral diaphragmatic paralysis - a rare cause of acute respiratory failure managed with nasal mask bilevel positive airway pressure (BiPAP) ventilation
}

\author{
M.C. Lin*, M.Y. Liaw**, C.C. Huang*, M.L. Chuang*, Y.H. Tsai*
}

Bilateral diaphragmatic paralysis - a rare cause of acute respiratory failure managed with nasal mask bilevel positive airway pressure (BiPAP) ventilation. M.C. Lin, M.Y. Liaw, C.C. Huang, M.L. Chuang, Y.H. Tsai. @ERS Journals Ltd 1997.

ABSTRACT: A $68 \mathrm{yr}$ old woman presented with acute respiratory failure. She was suspected of having a phrenic-diaphragmatic impairment, without evidence of an intrinsic lung disease or generalized neuromuscular disorder, after 3 weeks of prolonged mechanical ventilation.

A series of studies, including fluoroscopy, phrenic nerve stimulation test and diaphragmatic electromyography, was performed before the diagnosis of bilateral diaphragmatic paralysis (BDP) was confirmed. The patient was successfully weaned from the conventional mechanical ventilator, and was placed on nasal mask bi-level positive airway pressure (BiPAP) ventilation.

A high degree of clinical suspicion of bilateral diaphragmatic paralysis should always be raised in patients suffering respiratory failure without definite predisposing factors. Weaning with noninvasive nasal mask ventilation should be tried first instead of direct tracheostomy.

Eur Respir J 1997; 10: 1922-1924.
*Division of Pulmonary and Critical Care Medicine, Dept of Medicine, and **Dept of Rehabilitation Medicine, Chang Gung Memorial Hospital, Taipei, Taiwan, Republic of China.

Correspondence: M.C. Lin, Division of Pulmonary and Critical Care Medicine, Dept of Medicine, Chang Gung Memorial Hospital, 199 Tun-Hwa North Road, Taipei, Taiwan, Republic of China

Keywords: Acute respiratory failure diaphragmatic paralysis mask-ventilation mechanical ventilation

Received: September 191995 Accepted after revision April 291997
Bilateral diaphragmatic paralysis (BDP) is a rare disorder, which can be secondary to spinal cord injury [1], motor neuron disease, myopathy [2], noninfectious polyneuropathy, infection [3], iced saline cardioplegia performed during cardiac surgery [4], or idiopathic causes [3]. Although BDP has been well described, the condition is generally under-recognized, and diagnosis is frequently delayed, especially in a ventilator-dependent patient [5].

We report a patient who presented with acute respiratory insufficiency, without definite predisposing factors. Pulmonary, cardiac, infectious and neurological disorders were all excluded after detailed examination. The diagnosis of BDP was confirmed by meticulous survey. The patient benefited from nasal mask bi-level positive airway pressure (BiPAP; Respironics Inc., Murrysville, PA, USA), with excellent results. The necessity for a high degree of clinical suspicion is stressed, and the importance of choice to use noninvasive nasal mask ventilation to prevent tracheostomy is emphasized.

\section{Case report}

A $68 \mathrm{yr}$ old woman in relatively good health suffered from an episode of productive cough, fever and chills, 1 month before she attended our hospital. Symptoms subsided soon after taking drugs. General malaise and severe progressive dyspnoea, especially when supine, developed 3 days before she was admitted to our hospital. Profound respiratory failure occurred soon after her admission.
Initial arterial blood gas (ABG) values revealed: $\mathrm{pH}$ 7.19; arterial carbon dioxide tension $\left(\mathrm{Pa}_{\mathrm{a}} \mathrm{CO}_{2}\right) 10.5 \mathrm{kPa}$ $(79 \mathrm{mmHg})$, arterial oxygen tension $\left(\mathrm{Pa}_{\mathrm{a}} \mathrm{O}_{2}\right) 7.9 \mathrm{kPa}(59$ $\mathrm{mmHg}$ ) in room air. The patient was intubated and placed on mechanical ventilation for respiratory support. Her overall clinical condition gradually stabilized, but she could not be weaned from the ventilator after 3 weeks of ventilator support. Her repetitive weaning profile in spontaneous breathing showed a low tidal volume and low maximal inspiratory pressure (MIP), around $15 \mathrm{cmH}_{2} \mathrm{O}$. Chest, cardiac, infectious and primary neuromuscular disorders were all eliminated by a series of examinations, which led to the consideration of phrenicdiaphragmatic dysfunction.

Fluoroscopic examination showed paradoxical movement of bilateral diaphragmatic movement. A phrenic nerve conduction velocity (NCV) test, studied by transcutaneous phrenic nerve stimulation (Nicolet Viking II, 8 channel, as described by DAvis [6]), evoked no twitch in the diaphragm, and no diaphragmatic compound motor action potentials (CMAPs) were recorded. Diaphragmatic electromyography (EMG) and a peripheral nerve stimulation test confirmed bilateral diaphragmatic paralysis, without evidence of generalized neuromuscular disorders.

For trial of successful spontaneous breathing, the patient was placed on a BiPAP-spontaneous and timed $(\mathrm{S} / \mathrm{T})$ machine ( $\mathrm{S} / \mathrm{T}$ mode), using a nasal mask interface with an inspiratory positive airway pressure level (IPAP) of $16 \mathrm{cmH}_{2} \mathrm{O}$, an expiratory positive airway pressure (EPAP) of $4 \mathrm{cmH}_{2} \mathrm{O}$ and machine rate setting at 16 cycles. $\mathrm{min}^{-1}$ 
after extubation. The respiratory frequency was 16 breaths $\mathrm{min}^{-1}$, and $\mathrm{ABG}$ values were: $\mathrm{pH} 7.43, \mathrm{~Pa}_{\mathrm{a}} \mathrm{CO}_{2}$ $7.4 \mathrm{kPa}(56 \mathrm{mmHg}), P \mathrm{a}, \mathrm{O}_{2} 9.6 \mathrm{kPa}(72 \mathrm{mmHg})$, and arterial oxygen saturation $\left(\mathrm{Sa}_{2} \mathrm{O}_{2}\right) 94 \%$ on BiPAP ventilation and $\mathrm{O}_{2} 3 \mathrm{~L} \cdot \mathrm{min}^{-1}$. The patient was then able to sleep supine without breathing difficulties.

Two weeks later, the BiPAP machine was shifted to $\mathrm{S}$ mode. The IPAP was $10 \mathrm{cmH}_{2} \mathrm{O}$ and EPAP $4 \mathrm{cmH}_{2} \mathrm{O}$. The patient's respiratory frequency remained at approximately $12-18$ breaths $\cdot \mathrm{min}^{-1}$. She was discharged from hospital with full-day nasal mask BiPAP-S support and home oxygen therapy.

A further phrenic nerve conduction study 2 months later showed a detectable but decreased CMAP amplitude in the bilateral phrenic nerves. Measurement of motor and sensory nerve conduction revealed that only a decreased CMAP amplitude and slower NCV of the left ulnar nerve remained. MIP increased to $25 \mathrm{cmH}_{2} \mathrm{O}$. Spirometry was performed at the clinic, and showed forced vital capacity (FVC) $0.37 \mathrm{~L}$ ( $34 \%$ of predicted), forced expiratory volume in one second (FEV1) $0.32 \mathrm{~L}$ (40\% pred), and FEV1/FVC $82 \%$. At a 6 month followup, the patient remained on nasal mask BiPAP for 8 $\mathrm{h} \cdot \mathrm{day}^{-1}$ (during the night), and had an independent life.

\section{Discussion}

The patient's clinical symptoms and signs consisted of dyspnoea and paradoxical movement of the abdominal wall during inspiration, especially when in the supine position. Initially, the cause of acute respiratory failure was attributed either to sepsis or cardiac disorders. Neither could be confirmed by detailed examinations. Suspected diagnosis of BDP was not made until fluoroscopy was used to detect paradoxical diaphragmatic movement.

In addition to the clinical presentation, four methods were used to reach a diagnosis of BDP: 1) fluoroscopy; 2) spirometry; 3) phrenic nerve conduction tests; and 4) transdiaphragmatic pressure studies [7]. Many reports have stated that fluoroscopy may be misleading in BDP, because it can lead to incorrect results depending upon the patient's posture during examination [4, 8, 9]. In the upright position, active contraction of the abdominal muscles causes upward displacement of the paralysed diaphragm, which is followed by an abrupt termination of abdominal muscular activity in early inspiration, leading to a passive descent of the diaphragm $[2,10]$. This passive descent may be interpreted during fluoroscopy as evidence of active diaphragmatic contraction. Fluoroscopy was performed whilst the patient was in a supine position. Diaphragmatic movement was carefully examined: during spontaneously breathing (by sniff method); and passively by Ambu-resucitator (Ambu Inc., Linthicum, MD, USA) bagging (fig. 1).

Phrenic nerve stimulation and diaphragmatic EMG study are useful in demonstrating defects in nerve conduction, but in instances where no twitch can be evoked in the diaphragm, the possibility exists that this may simply be failure to locate the phrenic nerve, rather than absence of any diaphragmatic activity [10]. The assessment of diaphragmatic EMG provides a qualitative measure of the neuropathic and myopathic conditions of the diaphragm. Both tests were performed to confirm the diagnosis. No definite evidence of peripheral a)

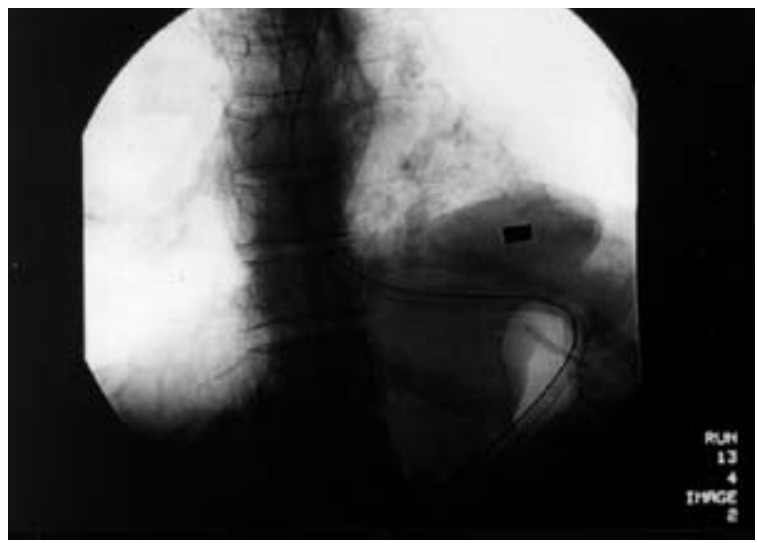

b)

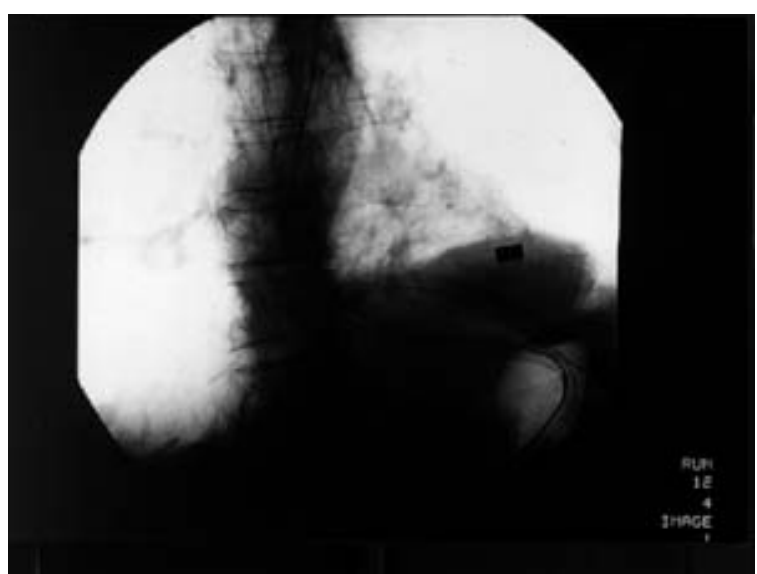

Fig. 1. - a) Bilateral diaphragmatic movement at the deep inspiration level of spontaneous breathing. Note the level of the marker below the left diaphragm. b) Insufflation by Ambu-bagging. The level of the left marker is lower than in spontaneous breathing. The patient is scoliotic, with the convex of the primary curve to the right.

neuromyopathy could be detected by peripheral NCV test and EMG. The follow-up phrenic nerve conduction study performed 2 months after discharge showed little improvement, which might explain why the patient was unable to leave BiPAP ventilation for a prolonged period.

The transdiaphragmatic pressure measurement provides a quantitative index of diaphragmatic contractility, because, when the diaphragm is paralysed, very little or no change in the transdiaphragmatic pressure occurs during an inspiratory manoeuvre [10]. To measure transdiaphragmatic pressure using a sniff manoeuvre, a sharp voluntary inspiratory effort through the nostrils with the mouth closed is an easy and helpful test in the diagnosis of BDP [11]. We did not perform this examination because no pressure transducer was available. Although MIP measurement was regarded as an indicator of global inspiratory muscle strength, one may still have to suspect significant diaphragmatic dysfunction if the data are abnormally low [12]. The MIP increased from $15 \mathrm{cmH}_{2} \mathrm{O}$ before discharge to 25 $\mathrm{cmH}_{2} \mathrm{O} 2$ months later, which might indicate the patient's increasing ability to reduce the duration of ventilatory support gradually.

The median delay in diagnosing BDP is reported to be 2 yrs, with a range of 6 weeks to 10 yrs [5]. The present patient suffered from clinical symptoms for 2 months until a definite diagnosis was made. It is worth 
mentioning that very few patients initially present with acute respiratory failure, especially when on mechanical ventilation. Without the development of "ventilatordependency" or "difficulty in weaning", the average length of delay has been substantially longer [5].

With respect to aetiology and prognosis, neuromuscular disease was the most commonly presumed condition responsible for phrenic-diaphragmatic impairment. Peripheral EMG and NCV tests were performed on the present patient to exclude the possibility of neuromuscular disorders. The prime cause for this patient's condition was presumed to be postinfection or idiopathic. It has been reported that patients developed diaphragmatic paralysis secondary to herpes zoster [3]. The present patient had symptoms of an upper respiratory tract infection 1 month before admission, but the exact relationship between that infection and BDP is not known.

Most patients with BDP have been treated by positive-pressure ventilators via tracheostomy [5, 7]. Other patients can live well under the support of negativepressure ventilators, such as cuirass or pneumobelt [5]. The present patient was extubated soon after the diagnosis was confirmed. Positive-pressure ventilation via nasal mask was chosen as a good method for ventilatory support, because the patient had only scanty secretion and was quite co-operative. The BiPAP ventilator was used instead of a conventional volume-cycled ventilator, because: 1) this patient still showed adequate drive for spontaneous breathing (tidal volume of $390 \mathrm{~mL}$ under $10 \mathrm{cmH}_{2} \mathrm{O}$ of ventilatory pressure support, with respiratory frequency 14 breaths $\mathrm{min}^{-1}$ before extubation); 2) with the BiPAP-S being used in continuous flow in contrast to the demand flow of a conventional volume-cycled ventilator, the work of breathing may be decreased; and 3) the cost of home care is lower. The patient currently leads an independent life with family support. The ventilation time has been decreased from $20 \mathrm{~h} \cdot \mathrm{day}^{-1}$ before discharge to $8 \mathrm{~h} \cdot \mathrm{day}^{-1}$ (at night, during sleep) at the present time.

A high degree of clinical suspicion of bilateral diaphragmatic paralysis should be raised, in patients with respiratory failure but without definite predisposing factors. For successful weaning, the physician may first choose noninvasive nasal mask ventilation instead of direct tracheostomy.

\section{References}

1. Glenn WWL, Hogan JF, Loke JSO, Ciesielski TE, Phelps ML, Rowedder R. Ventilatory support by pacing of conditioned diaphragm in quadriplegia. $N$ Engl $J$ Med 1984; 310: 1150-1155.

2. Newsom-Davis J. Diaphragm and neuromuscular disease. Am Rev Respir Dis 1979; 119 (2): 115-117.

3. Dutt AK. Diaphragmatic paralysis caused by herpes zoster. Am Rev Respir Dis 1970; 101: 755-758.

4. Kohorst WR, Schonfeld SA, Altman M. Bilateral diaphragmatic paralysis following topical cardiac hypothermia. Chest 1984; 85: 65-68.

5. Chan CK, Loke J, Virgulto JA, Mohsenin V, Ferranti, Lammertse T. Bilateral diaphragmatic paralysis: clinical spectrum, prognosis and diagnostic approach. Arch Phys Med Rehabil 1988; 69: 976-979.

6. Davis JN. Phrenic nerve conduction in man. J Neurol Neurosurg Psychiatry 1967; 30: 420-426.

7. Brown K, Hoffstein V, Byrick R. Bedside diagnosis of bilateral diaphragmatic paralysis in a ventilator-dependent patient after open-heart surgery. Anesth Analg 1985; 64: 1208-1210.

8. Chandler K, Rozas C, Kory R, Goldman A. Bilateral diaphragmatic paralysis complicating local cardiac hypothermia during open heart surgery. Am J Med 1984; 77: 243-249.

9. Schiavi E. Isolated bilateral diaphragmatic paresis with interstitial lung disease. Am Rev Respir Dis 1984; 129: 337-339.

10. Loh L, Goldman M, Newsom Davis J. Assessment of diaphragm function. Medicine 1977; 56: 165-169.

11. Miller J, Moxham J, Green M. The maximal sniff in the assessment of diaphragm function in man. Clin Sci 1985; 69: 91-96.

12. Rochester DF, Arora NS. Respiratory muscle failure. Med Clin North Am 1983; 67: 573-597. 